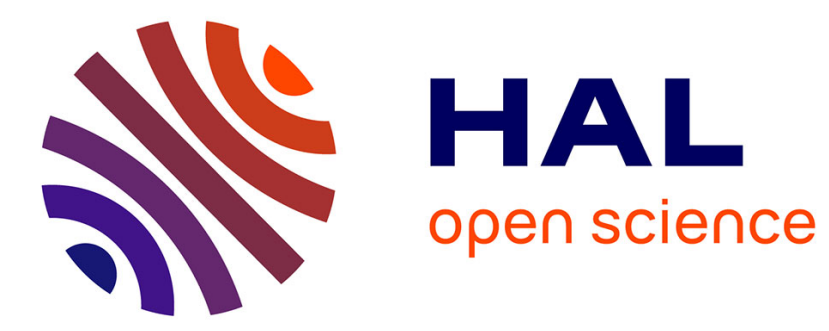

\title{
A novel approach to the automatic control of scale model airplanes
}

Minh-Duc Hua, Daniele Pucci, Tarek Hamel, Pascal Morin, Claude Samson

\section{To cite this version:}

Minh-Duc Hua, Daniele Pucci, Tarek Hamel, Pascal Morin, Claude Samson. A novel approach to the automatic control of scale model airplanes. 53rd IEEE Conference on Decision and Control, Dec 2014, Los Angeles, United States. pp.805 - 812, 10.1109/CDC.2014.7039480 . hal-01377807

\section{HAL Id: hal-01377807 https://hal.science/hal-01377807}

Submitted on 11 Oct 2016

HAL is a multi-disciplinary open access archive for the deposit and dissemination of scientific research documents, whether they are published or not. The documents may come from teaching and research institutions in France or abroad, or from public or private research centers.
L'archive ouverte pluridisciplinaire HAL, est destinée au dépôt et à la diffusion de documents scientifiques de niveau recherche, publiés ou non, émanant des établissements d'enseignement et de recherche français ou étrangers, des laboratoires publics ou privés. 


\title{
A novel approach to the automatic control of scale model airplanes
}

\author{
Minh-Duc Hua ${ }^{1}$, Daniele Pucci ${ }^{2}$, Tarek Hamel ${ }^{3}$, Pascal Morin ${ }^{1}$, Claude Samson ${ }^{3,4}$ \\ 1 ISIR CNRS-UPMC, Paris, France, hua@isir.upmc.fr, morin@isir.upmc.fr \\ 2 Italian Institute of Technology, Genova, Italy, daniele.pucci@iit.it \\ 3 I3S/UNSA, Sophia-Antipolis, France, thamel@i3s.unice.fr \\ 4 INRIA, I3S/UNSA, Sophia Antipolis, France, claude.samson@inria.fr, csamson@i3s.unice.fr
}

\begin{abstract}
This paper explores a new approach to the control of scale model airplanes as an extension of previous studies addressing the case of vehicles presenting a symmetry of revolution about the thrust axis. The approach is intrinsically nonlinear and, with respect to other contributions on aircraft nonlinear control, no small attack angle assumption is made in order to enlarge the controller's operating domain. Simulation results conducted on a simplified, but not overly simplistic, model of a small airliner illustrate the soundness of the approach.
\end{abstract}

Keywords: aerial vehicles, fixed-wing aircraft, dynamics of flight, nonlinear feedback control

\section{INTRODUCTION}

The present paper explores a new approach to the nonlinear control of small underactuated aerial vehicles equipped with fixed planar wings, alike common scale model airplanes. Besides purely scientific issues, this research is motivated by the emerging and rapidly growing market of small automatized aerial drone-type vehicles ranging from VTOL devices (helicopters, quadrotor vehicles,...) to small cruising airplanes used for a multitude of applications (site surveillance, rescue missions, landscape mapping, aerial photography,...). Controlling these devices calls for a unifying theory which is both generic and adaptable to the specificities of each vehicle. As for the usefulness of a nonlinear control approach, it partly resides in the fact that small airplanes are particularly sensitive to wind perturbations and thus confronted to flight conditions involving large and rapidly varying attack angles that existing autopilots, based on linear control techniques, cannot handle properly.

The proposed approach departs from traditional approaches based on the linearisation of the vehicle's dynamics about a number of operating conditions and developed primarily for full-size commercial aerial vehicles (airplanes and helicopters) meant to fly along specific trajectories (trim trajectories) that involve a narrow range of attack angles. Many aerial vehicles are required to fly in more diverse conditions that involve large and rapid variations of the attack angle. Examples are given by fighter aircraft, convertible aircraft, or small Unmanned Aerial vehicles (UAVs) operating in windy environments. Vertical Take-Off and Landing (VTOL) vehicles, like e.g. ducted fans and 3D scale model airplanes, are also subjected to large variations of the attack angle when transitioning from hover to horizontal cruising flight. For the automatic control of these devices it matters to ensure a large stability domain that is provably achievable only with a nonlinear control design. Nonlinear feedback control of aircraft can be traced back to the early eighties. Following [13], control laws based on the dynamic inversion technique have been proposed to extend the flight envelope of military aircraft (see, e.g., [14] and the references therein). The control design strongly relies on tabulated models of aerodynamic forces and moments, like the High-Incidence Research Model (HIRM) of the Group for Aeronautical Research and Technology in Europe (GARTEUR) [9]. However, the attack angle is assumed to remain small and away from the stall zone. Should this assumption be violated the system's behavior is unpredictable. Comparatively, nonlinear feedback control of VTOL vehicles is more recent and has been addressed with a larger variety of techniques. Besides dynamic inversion [3], other techniques include Lyapunovbased design [8], [6], Backstepping [2], Sliding modes [2], [15], and Predictive control [7], [1]. A more complete bibliography on this topic is provided in [4]. However, most of these studies address the stabilization of hover flight or of low-velocity trajectories, and little attention is paid to aerodynamic effects. These are typically either ignored or modelled as a simple additive perturbation the effect of which has to be compensated for by the feedback action. For highly dynamic flight, as in the case of cruising airplanes taking advantage of aerodynamic lift, or in harsh wind conditions, aerodynamic effects cannot be neglected. A proper modelling of aerodynamic effects for the purpose of designing feedback controllers that perform well in a large flight domain, and the design of such controllers whose performance is supported by mathematical analysis are issues that have been seldom be addressed so far by the control and robotics communities. An important difficulty comes from the fact that classical methods used in aerodynamic modelling to precisely describe aerodynamic forces, e.g. computational fluid dynamics (CFD) or wind tunnel measurements, do not provide analytical expressions of aerodynamic characteristics. From a control design perspective these methods are useful to finely tune a controller around a given flight velocity. But they are not best suited to handle the case of large flight envelopes that involve strong variations of either the flight velocity or the attack angle. We instead advocate the use of simple analytical models of aerodynamic characteristics. Although relatively 
imprecise, these models account for important structural properties of the system in a large flight envelope. The idea behind our approach is to exploit these properties at the control design level and rely on the robustness of feedback controllers to cope with discrepancies between the model and the true aerodynamic characteristics.

The control design here proposed follows the one reported in [12] and extends it to the case of fixed-wing aircraft whose shape satisfies a symmetry property weaker than the axisymmetry property considered in this previous work. In particular, we show how the stabilization of the airplane's sideslip angle via roll control -a control which is not needed in the axisymmetric case to track reference (velocity or position) trajectories, but with which every airplane's human pilot is familiar- brings the control problem back to the one of the axisymmetric case for the determination of the other control variables.

The paper is organized as follows. Section II provides notation and background material. The main results are reported in Section III. First, structural properties of the aerodynamic force induced by the symmetry of the airplane are pointed out and an expression of this force, depending essentially on the attack angle when the sideslip angle is equal to zero, is worked out. We then show how the sideslip angle can be stabilized at zero via roll feedback control. Zeroing this angle achieves what is commonly called the coordinated flight between roll and yaw control actions. Once the sideslip angle vanishes, the aerodynamic force applied to the aircraft is similar to the one applied to an axisymmetric vehicle, and the other control variables can be determined according to the approach presented in [12]. The control design is complemented with a convergence and stability result for a class of reference trajectories. In Section IV simulations performed on a simplified, but nonetheless physically credible, model of a small AUV illustrate the robust performance of the proposed controller. Concluding remarks and perspectives are proposed in Section V.

\section{BACKGROUND AND NOTATION}

\section{A. Notation}

Throughout the paper, $\boldsymbol{E}^{3}$ denotes the $3 D$ Euclidean vector space and vectors in $\boldsymbol{E}^{3}$ are denoted with bold letters. Inner and cross products in $\boldsymbol{E}^{3}$ are denoted by $\cdot$ and $\times$ respectively. Let us consider a rigid body immersed in air, as represented by Fig. 1. The following notation is used.

- $G$ denotes the body's center of mass (CoM);

- $\mathcal{I}=\left\{O ; \boldsymbol{i}_{0}, \boldsymbol{j}_{0}, \boldsymbol{k}_{0}\right\}$ is an inertial frame;

- $\mathcal{B}=\{G ; \boldsymbol{i}, \boldsymbol{j}, \boldsymbol{k}\}$ is a body-fixed frame;

- $\boldsymbol{\omega}$ is the angular velocity of the body-fixed frame with respect to (w.r.t.) the inertial frame;

- $m$ is the body's mass;

- $v$ is the CoM's velocity w.r.t. the inertial frame;

- $\boldsymbol{a}$ is the CoM's acceleration w.r.t. the inertial frame;

- $\boldsymbol{g}=g \boldsymbol{k}_{0}$ is the gravitational acceleration;

- $\boldsymbol{v}_{a}=\boldsymbol{v}-\boldsymbol{v}_{w}$ is the body's air-velocity (i.e., translational velocity w.r.t. the ambient air), with $\boldsymbol{v}_{w}$ denoting the wind's velocity. The direction of $\boldsymbol{v}_{a}$ in the body-fixed frame is often represented by the two angles $\alpha$ and $\beta$ denoting respectively the attack angle and sideslip angle, i.e.

$$
\boldsymbol{v}_{a}=\left|\boldsymbol{v}_{a}\right|(\sin \alpha \boldsymbol{i}+\cos \alpha(\sin \beta \boldsymbol{j}-\cos \beta \boldsymbol{k}))
$$

This definition of attack and sideslip angles slightly differs from the one classically used in aeronautics, but it is locally equivalent when these angles are small. Note also that, alike any parametrization of the unit sphere by two angles, this representation is not uniquely defined everywhere. More precisely, $\beta$ cannot be defined by continuity at $\alpha= \pm \pi / 2$, nor when $\left|\boldsymbol{v}_{a}\right|=0$.

The coordinate vector of any $\boldsymbol{\xi} \in \boldsymbol{E}^{3}$ w.r.t. the body-fixed frame $\mathcal{B}$ is denoted by the ordinary letter $\xi$, i.e. $\boldsymbol{\xi}=\xi_{1} \boldsymbol{i}+$ $\xi_{2} \boldsymbol{j}+\xi_{3} \boldsymbol{k}$. Throughout the paper, either $\dot{\boldsymbol{\xi}}$ or $\frac{d}{d t} \boldsymbol{\xi}$ denotes the time-derivative of $\boldsymbol{\xi} \in \boldsymbol{E}^{3}$ relatively to the inertial frame $\mathcal{I}$.

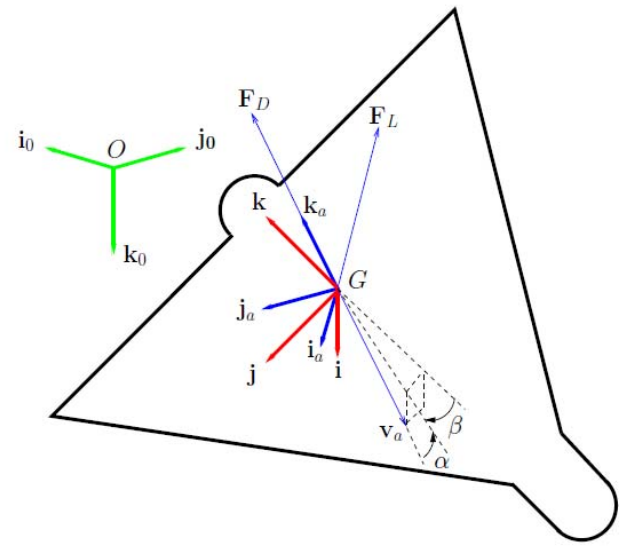

Fig. 1. Sketch representation of a scale model airplane

\section{B. Aerodynamic forces}

The resultant aerodynamic force $\boldsymbol{F}_{a}$ applied to a rigid body moving with air-velocity $\boldsymbol{v}_{a}$ is traditionally decomposed into the sum of a drag force $\boldsymbol{F}_{D}$ along the direction of $\boldsymbol{v}_{a}$ and a lift force $\boldsymbol{F}_{L}$ perpendicular to this direction, i.e.

$$
\boldsymbol{F}_{a}=\boldsymbol{F}_{D}+\boldsymbol{F}_{L}
$$

The intensities of drag and lift forces are essentially proportional to $\left|\boldsymbol{v}_{a}\right|^{2}$ modulo variations characterized by two dimensionless functions $C_{D}$ and $C_{L}$, which depend in the first place on the orientation of $\boldsymbol{v}_{a}$ w.r.t. the body, but also on the Reynolds number $R_{e}$ and Mach number $M$. These dimensionless functions are called the aerodynamic characteristics of the body, or drag coefficient and lift coefficient respectively. More precisely

$$
\boldsymbol{F}_{D}=-\eta_{a}\left|\boldsymbol{v}_{a}\right| C_{D} \boldsymbol{v}_{a}, \quad \boldsymbol{F}_{L}=\eta_{a}\left|\boldsymbol{v}_{a}\right| C_{L} \boldsymbol{v}_{a}^{\perp}
$$

with

- $\boldsymbol{v}_{a}^{\perp}$ a vector perpendicular to $\boldsymbol{v}_{a}$ and with same norm,

- $\eta_{a}:=\frac{\rho \Sigma}{2}$ with $\rho$ the free stream air density, and $\Sigma$ an area germane to the body shape.

Another expression of drag and lift forces can be obtained by introducing an air-frame $\mathcal{A}=\left\{G ; \boldsymbol{i}_{a}, \boldsymbol{j}_{a}, \boldsymbol{k}_{a}\right\}$ defined as follows (See Fig. 1): 


$$
\boldsymbol{k}_{a}=-\frac{\boldsymbol{v}_{a}}{\left|\boldsymbol{v}_{a}\right|}, \quad \boldsymbol{i}_{a}=-\frac{\boldsymbol{v}_{a}^{\perp}}{\left|\boldsymbol{v}_{a}\right|}, \quad \boldsymbol{j}_{a}=\boldsymbol{k}_{a} \times \boldsymbol{i}_{a}
$$

Then (3) can also be written as

$$
\boldsymbol{F}_{D}=\eta_{a}\left|\boldsymbol{v}_{a}\right|^{2} C_{D} \boldsymbol{k}_{a}, \quad \boldsymbol{F}_{L}=-\eta_{a}\left|\boldsymbol{v}_{a}\right|^{2} C_{L} \boldsymbol{i}_{a}
$$

Throughout the paper we assume that the aerodynamic characteristics and the lift-force direction (i.e., the vector $\boldsymbol{i}_{a}$ ) depend on the direction of $\boldsymbol{v}_{a}$ but not on its intensity. We also neglect the influence of Reynolds and Mach numbers variations on these functions. In other words, $C_{D}=$ $C_{D}\left(\boldsymbol{k}_{a}\right), C_{L}=C_{L}\left(\boldsymbol{k}_{a}\right)$, and $\boldsymbol{i}_{a}=\boldsymbol{i}_{a}\left(\boldsymbol{k}_{a}\right)$. As commonly done in the dynamics of flight literature, we may also write, with a slight abuse of notation, $C_{D}=C_{D}(\alpha, \beta)$, $C_{L}=C_{L}(\alpha, \beta)$ when the attack and sideslip angles are well defined.

\section{Control problem statement}

The control objective here chosen is the asymptotic stabilization of either a desired (or reference) velocity $\boldsymbol{v}_{r}$ or a desired position $\boldsymbol{p}_{r}$ for the vehicle's CoM. It is assumed that the control inputs consist of a thrust force $\boldsymbol{T}=-T \boldsymbol{k}$, with $T$ denoting the thrust intensity, and a torque vector $\Gamma$, independent of the thrust actuation, used to modify the body's angular velocity $\boldsymbol{\omega}$-and thus the body's orientation. Nominally the resultant thrust force passes near the center of mass and thus exerts a small parisitic torque. Aerodynamic forces that apply to the body may also create a resultant parasitic torque $\Gamma_{a}$. The control torque $\Gamma$ then has to precompensate for these parasitic torques and also ensure that (almost) any desired angular velocity is obtained rapidly (instantaneously, in the ideal case). This leads to consider the angular velocity $\boldsymbol{\omega}$ as an intermediate control input, with the conceptually important advantage of not having to take the specificities of the physical torque actuation at this stage of the control design. This corresponds to a standard "backstepping" assumption. Once it is made, the vehicle's actuation consists of four input variables, namely, the thrust intensity and the three coordinates of $\boldsymbol{\omega}$.

The control model then reduces to the classical Newton's equation complemented with the orientation kinematics equation:

$$
\begin{aligned}
m \dot{\boldsymbol{v}} & =m \boldsymbol{g}+\boldsymbol{F}_{a}-T \boldsymbol{k} \\
\frac{d}{d t}(\boldsymbol{i}, \boldsymbol{j}, \boldsymbol{k}) & =\boldsymbol{\omega} \times(\boldsymbol{i}, \boldsymbol{j}, \boldsymbol{k})
\end{aligned}
$$

\section{MAIN RESUlTS}

\section{A. Modelling of aerodynamic forces for control design}

The following way of writing the aerodynamic force is instrumental for the proposed control approach.

Proposition 1 Assume that $k_{a, 1} \neq 0$. Then, the aerodynamic force can be expressed as

$$
\boldsymbol{F}_{a}=-\eta_{a}\left|\boldsymbol{v}_{a}\right|\left[\left(C_{D}-C_{L} \frac{i_{a, 1}}{k_{a, 1}}\right) \boldsymbol{v}_{a}-\frac{C_{L}\left|\boldsymbol{v}_{a}\right|}{k_{a, 1}} \boldsymbol{i} \times \boldsymbol{j}_{a}\right]
$$

Proof: Recall the classical vectorial relation:

$$
\forall \boldsymbol{x}, \boldsymbol{y}, \boldsymbol{z} \in \boldsymbol{E}^{3}, \quad \boldsymbol{x} \times(\boldsymbol{y} \times \boldsymbol{z})=(\boldsymbol{x} \cdot \boldsymbol{z}) \boldsymbol{y}-(\boldsymbol{x} \cdot \boldsymbol{y}) \boldsymbol{z}
$$

Since $\boldsymbol{j}_{a}=\boldsymbol{k}_{a} \times \boldsymbol{i}_{a}$, it follows from (8) that

$$
\begin{aligned}
\boldsymbol{i} \times \boldsymbol{j}_{a} & =\left(\boldsymbol{i} \cdot \boldsymbol{i}_{a}\right) \boldsymbol{k}_{a}-\left(\boldsymbol{i} \cdot \boldsymbol{k}_{a}\right) \boldsymbol{i}_{a} \\
& =i_{a, 1} \boldsymbol{k}_{a}-k_{a, 1} \boldsymbol{i}_{a}
\end{aligned}
$$

Therefore,

$$
\boldsymbol{i}_{a}=\frac{1}{k_{a, 1}}\left(i_{a, 1} \boldsymbol{k}_{a}-\boldsymbol{i} \times \boldsymbol{j}_{a}\right)
$$

and (7) readily follows from (2) and (5).

Proposition 1 holds independently of the vehicle's shape. In order to further specify aerodynamic forces, we introduce an assumption that is satisfied for almost all aerial vehicles.

Assumption 1 The vehicle is symmetric with respect to the plane $\{G, \boldsymbol{i}, \boldsymbol{k}\}$, i.e.

$$
(x \boldsymbol{i}+y \boldsymbol{j}+z \boldsymbol{k}) \in \mathcal{S} \Longrightarrow(x \boldsymbol{i}-y \boldsymbol{j}+z \boldsymbol{k}) \in \mathcal{S}
$$

with $\mathcal{S}$ the vehicle's surface.

For control design purposes, it is useful to have a more precise expression of the lateral component $F_{a, 2}$ of the aerodynamic force, because this component determines the variations of the lateral velocity component $v_{a, 2}$, and thus the variations of the sideslip angle $\beta$. The following proposition provides such an expression under mild assumptions.

\section{Proposition 2 Assume that}

1) $C_{L}$ is a smooth function of $(\alpha, \beta)$ around $\alpha=0$ and $C_{L}(0, \beta)=0 \forall \beta$;

2) The vector $\boldsymbol{i}_{a}$ varies smoothly with $\boldsymbol{k}_{a}$;

Then, the following relationship is satisfied:

$$
F_{a, 2}=-a\left(k_{a}\right)\left|\boldsymbol{v}_{a}\right| v_{a, 2}+b\left(k_{a}\right) v_{a, 1} v_{a, 2}
$$

with $a(\cdot)$ and $b(\cdot)$ denoting continuous functions, and $a(\cdot)$ strictly positive.

The proof of this proposition is given in the appendix.

Remark: The first condition in Proposition 2 is satisfied when the plane $\{G, \boldsymbol{j}, \boldsymbol{k}\}$, which is orthogonal to the plane of symmetry evoked in Assumption 1 and to which the thrust direction is parallel, corresponds to the zero lift-plane, i.e., $\alpha=0 \Longrightarrow \boldsymbol{F}_{L}=0$. As for the second (regularity) condition, one can reasonably expect that it is satisfied in practice, even though a rigorous mathematical justification remains to be worked out.

Another important consequence of the vehicle's symmetry is that if $\boldsymbol{v}_{a}$ belongs to the plane $\{G, \boldsymbol{i}, \boldsymbol{k}\}$, i.e. if the sideslip angle is zero, then the lift force $\boldsymbol{F}_{L}$ also belongs to this plane. Also $\boldsymbol{j}_{a}=\boldsymbol{j}, \boldsymbol{k}_{a}=-\sin \alpha \boldsymbol{i}+\cos \alpha \boldsymbol{k}$, and one can set $\boldsymbol{i}_{a}=\cos \alpha \boldsymbol{i}+\sin \alpha \boldsymbol{k}$ since $\boldsymbol{i}_{a}$ is orthogonal to $\boldsymbol{k}_{a}$ and belongs to the plane $\{G, \boldsymbol{i}, \boldsymbol{k}\}$ in this case.

Let $\boldsymbol{F}_{a}^{0}$ denote the aerodynamic force when $\beta=0$. In view of (7) and the above relations one deduces that

$$
\boldsymbol{F}_{a}^{0}=-\eta_{a}\left|\boldsymbol{v}_{a}\right|\left[\left(C_{D}^{0}+C_{L}^{0} \cot \alpha\right) \boldsymbol{v}_{a}+\frac{C_{L}^{0}\left|\boldsymbol{v}_{a}\right|}{\sin \alpha} \boldsymbol{k}\right]
$$

where the ${ }^{0}$ superscript denotes evaluation at $\beta=0$, i.e., $C_{D}^{0}(\alpha)=C_{D}(\alpha, 0), C_{L}^{0}(\alpha)=C_{L}(\alpha, 0)$. 
It then matters, for control purposes, to know more about the lift and drag coefficients and, in particular, on the way they depend on the attack angle. For small angles, a common model consists of choosing $C_{D}^{0}$ constant and $C_{L}^{0}$ a linear function of the attack angle, i.e. $\left(C_{D}^{0}=c_{0}, C_{L}^{0}=2 c_{1} \alpha\right)$ for some positive numbers $c_{0}$ and $c_{1}$. With these coefficients one readily verifies that the aerodynamic force $\boldsymbol{F}_{a}^{0}$ of relation (10) is, in the first approximation, equal to

$$
\boldsymbol{F}_{a}^{0}=-\eta_{a}\left|\boldsymbol{v}_{a}\right|\left(\bar{c}_{0} \boldsymbol{v}_{a}-2 c_{1} v_{a, 3} \boldsymbol{k}\right)
$$

with $\bar{c}_{0}=c_{0}+2 c_{1}$, when $\alpha$ is small. The above expression of the aerodynamic force is remarkable because it indicates that this force is the sum of two terms: one along the air velocity $\boldsymbol{v}_{a}$ and independent of the vehicle's orientation, and the other one along the thrust direction. For control purposes, these are good terms. Indeed, since the first term is independent of the vehicle's orientation, the problem of aligning the vehicle's thrust direction with it via the angular velocity control $\boldsymbol{\omega}$ is a well posed problem (see, for instance, [5] or [12] for an (almost) globally exponentially stabilizing solution). As for the second term that is aligned with the thrust direction, it can be compensated for directly via a suitable choice of the thrust intensity $T$. This is an important remark because it explains how orientation control and thrust control can be decoupled and, to some extent, why human pilots are capable of manually controlling airplanes without the help of control and stability augmentation systems (CSAS).

Now, in order to address situations when the attack angle is not small one needs expressions of the drag and lift coefficients over the whole range of possible angles. These expressions have to be compatible with the choice pointed out before for small angles, and they have to be reasonably representative of the physical reality. It is also useful to observe that, in order to preserve the property of independence w.r.t. the vehicle's orientation of the first term of $\boldsymbol{F}_{a}^{0}$, a sufficient condition is the invariance of $\left(C_{D}^{0}(\alpha)+C_{L}^{0}(\alpha) \cot \alpha\right)$ w.r.t. the attack angle. Examples of functions that satisfy this condition in a large domain of attack angles and are compatible with measurements performed on NACA wing profiles and axisymmetric shapes are reported in [10] and [12]. The most important limitation comes from the stall phenomenon corresponding to the brutal reduction of lift occurring beyond a certain value of the attack angle and in a (usually) narrow range of this angle (the stall zone). In the stall zone, the aforementioned invariance condition cannot be satisfied.

For the sake of simplifying the control design exposition, we will consider from now on the particular set of functions specified by the following assumption:

Assumption 2 The vehicle's aerodynamic characteristics $C_{D}, C_{L}$ are such that:

$$
C_{D}^{0}(\alpha)=c_{0}+2 c_{1} \sin ^{2} \alpha, \quad C_{L}^{0}(\alpha)=c_{1} \sin 2 \alpha
$$

with $c_{0}$ and $c_{1}$ two positive numbers.

These functions are $\pi$-periodic and respectively even and odd w.r.t. $\alpha$, they generalize the linear approximations evoked previously, and they satisfy the invariance condition since one readily verifies that $C_{D}^{0}(\alpha)+C_{L}^{0}(\alpha) \cot \alpha=c_{0}+2 c_{1}(=$ $\left.\bar{c}_{0}\right)$. These properties are consistent with the aerodynamic characteristics of a body shape symmetric with respect to both the planes $\{G, \boldsymbol{j}, \boldsymbol{k}\}$ and $\{G, \boldsymbol{i}, \boldsymbol{j}\}$, as in the case of NACA00XX wing profiles. For more general body shapes, $C_{D}^{0}$ and $C_{L}^{0}$ as defined by (12) can still be used as an approximation of many body aerodynamic characteristics in a large flight domain [10] [12]. The aerodynamic force expression, when the sideslip angle is kept equal to zero, is then given by (11). On the basis of this expression, and of the expression (9) of the lateral component of the aerodynamic force, the control design that we propose consists in $i$ ) controlling the sideslip angle to zero via the control input $\omega_{3}$, and $i$ ) monitoring the control thrust force via $T$ and $\omega_{1,2}$ so as to compensate for $\boldsymbol{F}_{a}^{0}$-complemented with the force of gravity- and tracking errors.

\section{B. Sideslip angle control}

We assume from now on that the wind's velocity is constant w.r.t. the inertial frame, i.e. $\dot{\boldsymbol{v}}_{w} \equiv 0$. Since $v_{a}$ is the coordinate vector of $\boldsymbol{v}_{a}$ in body-frame, it follows from (6) that

$$
m \dot{v}_{a}=m v_{a} \times \omega+m g+F_{a}-T e_{3}
$$

The control problem of the sideslip angle to zero is equivalent to controlling $v_{a, 2}$ to zero. Then,

$$
\dot{v}_{a, 2}=v_{a, 3} \omega_{1}-v_{a, 1} \omega_{3}+g_{2}+\frac{1}{m} F_{a, 2}
$$

Using the expression (9) of $F_{a, 2}$ given in Proposition 2, this equation can be written as

$$
\dot{v}_{a 2}=v_{a, 3} \omega_{1}-v_{a, 1} \omega_{3}+g_{2}-\frac{1}{m}\left(a\left|v_{a}\right| v_{a, 2}-b v_{a, 1} v_{a, 2}\right)
$$

If we calculate the third component of the angular velocity as follows

$$
\omega_{3}=\frac{v_{a, 3} \omega_{1}+g_{2}}{v_{a, 1}}+\mu \operatorname{sign}\left(v_{a, 1}\right) v_{a, 2}
$$

with $\mu \geq 0$ (not necessarily constant), then the resulting closed-loop equation is

$$
\dot{v}_{a, 2}=-\left(\frac{a}{m}\left|v_{a}\right|-\frac{b}{m} v_{a, 1}+\mu\left|v_{a, 1}\right|\right) v_{a, 2}
$$

which implies the asymptotic stability of $v_{a, 2}=0$ with an exponential rate of convergence to zero, provided that $\mu \geq \frac{b}{m}$ and $\left|v_{a, 1}\right|$ is strictly larger than a positive number. The control expression (14) calls for a few remarks and adaptations. This control is defined provided that $v_{a, 1}$, and thus the attack angle $\alpha$, is different from zero. While this condition is generically satisfied during a "standard" cruising flight, the attack angle may pass through zero during acrobatic manœuvres or in perturbed airflows. Assuming that the vehicle can hover at zero velocity without wind, then the air velocity is also zero and division by $v_{a, 1}$ is again ill-defined. The corresponding physical problem is that roll modification has no instantaneous influence of the sideslip angle when the attack angle vanishes. It is then better to impose a zero roll control. Note also that the problematic term in (14) is the pre-compensation term $\left(v_{a, 3} \omega_{1}+g_{2}\right) / v_{a, 1}$. 
In practice it may be sufficient to keep only the feedback part of the control expression, with a large enough gain $\mu$. This is basically what a human pilot does when using a balance indicator (inclinometer), or a "turn-and-slip indicator", to achieve "balanced flight". Another possibility consists in using a term that imperfectly pre-compensates for the term $\left(v_{a, 3} \omega_{1}+g_{2}\right)$ in (13), but is always well defined. For example, instead of (14), one may consider the following continuous control expression

$$
\omega_{3}=\frac{v_{a, 1}}{v_{a, 1}^{2}+\epsilon}\left(v_{a, 3} \omega_{1}+g_{2}\right)+\mu \frac{v_{a, 1}}{\left|v_{a, 1}\right|+\epsilon} v_{a, 2}
$$

with $\epsilon>0$ a small constant.

\section{Thrust control}

Let us assume for the time being, and for the sake of simplifying the presentation of the control design, that roll control is active and fulfills its role of keeping the sideslip angle $\beta$ equal to zero. Then $\boldsymbol{F}_{a}=\boldsymbol{F}_{a}^{0}$, as specified by (11). Consider a reference velocity $\boldsymbol{v}_{r}$, which may be the time derivative of a reference position $\boldsymbol{p}_{r}$, and denote the reference acceleration w.r.t. the inertial frame as $\boldsymbol{a}_{r}:=\frac{d}{d t} \boldsymbol{v}_{r}$. Velocity and position errors are respectively denoted as $\tilde{\boldsymbol{v}}:=\boldsymbol{v}-\boldsymbol{v}_{r}$ and $\tilde{\boldsymbol{p}}:=\boldsymbol{p}-\boldsymbol{p}_{r}$. From Newton's equation (6) one deduces

$$
m \dot{\tilde{\boldsymbol{v}}}=-T \boldsymbol{k}+m\left(\boldsymbol{g}-\boldsymbol{a}_{r}\right)+\boldsymbol{F}_{a}^{0}
$$

Using (11), this equation can in turn be written as

$$
m \dot{\tilde{\boldsymbol{v}}}=-T_{p} \boldsymbol{k}+\boldsymbol{F}_{p}
$$

with $T_{p}:=T-2 c_{1} \eta_{a}\left|\boldsymbol{v}_{a}\right| v_{a, 3}$ and $\boldsymbol{F}_{p}:=-\eta_{a} \bar{c}_{0}\left|\boldsymbol{v}_{a}\right| \boldsymbol{v}_{a}+$ $m\left(\boldsymbol{g}-\boldsymbol{a}_{r}\right)$. The main interest of this writing is that $\boldsymbol{F}_{p}$ does not depend on the vehicle's orientation. This equation is similar to the one derived in [12] for axisymmetric vehicles. It is also formally the same as the equation of a spherical body subjected to an aerodynamic force reduced to the drag component $-\eta_{a} \bar{c}_{0}\left|\boldsymbol{v}_{a}\right| \boldsymbol{v}_{a}$. This is what we have called spherical equivalence. Once it is established, the control design essentially reduces to transposing the control laws derived for the simpler spherical case.

Let $(\tilde{\boldsymbol{\rho}}, \tilde{\boldsymbol{v}})$ denote a "generalized" error vector. The precise definition of $\tilde{\boldsymbol{\rho}}$ depends on the control objective. For instance, it can be equal to $\tilde{\boldsymbol{p}}$, if the objective is reference position tracking, or it can be an integral of the velocity error $\tilde{\boldsymbol{v}}$, if the control objective is reference velocity tracking and an integral correction term is needed to improve the control performance. In the case of reference position tracking it may also be useful to introduce an integral correction. Then $\tilde{\boldsymbol{\rho}}=\left(\boldsymbol{I}_{p}, \tilde{\boldsymbol{p}}\right)$, with $\boldsymbol{I}_{p}$ denoting a saturated integral of the position tracking error. The simplest case corresponds to pure velocity control without integral correction, for which $\tilde{\boldsymbol{\rho}}=\emptyset$.

The idea is to transform the initial control problem into the much simpler one associated with the linear control system $\dot{\tilde{\boldsymbol{v}}}=\boldsymbol{\xi}$, with $\boldsymbol{\xi}$ denoting an intermediary feedback control input in charge of ensuring the exponential stability of the generalized error vector $(\tilde{\boldsymbol{\rho}}, \tilde{\boldsymbol{v}})$ to zero for this system. In view of (17), the relation $\tilde{\boldsymbol{v}}=\boldsymbol{\xi}$ can be satisfied if and only if $T_{p} \boldsymbol{k}=\overline{\boldsymbol{F}}_{p}$ with

$$
\overline{\boldsymbol{F}}_{p}:=\boldsymbol{F}_{p}-m \boldsymbol{\xi}
$$

This in turn implies that $T_{p}=\overline{\boldsymbol{F}}_{p} \cdot \boldsymbol{k}$, so that

$$
\begin{aligned}
T & =\left(\boldsymbol{F}_{a}^{0}+m\left(\boldsymbol{g}-\boldsymbol{a}_{r}-\boldsymbol{\xi}\right)\right) \cdot \boldsymbol{k} \\
& =-\eta_{a} c_{0}\left|\boldsymbol{v}_{a}\right| v_{a 3}+m\left(\boldsymbol{g}-\boldsymbol{a}_{r}-\boldsymbol{\xi}\right) \cdot \boldsymbol{k},
\end{aligned}
$$

Also $\boldsymbol{k}$ should be equal to $\boldsymbol{k}_{r}:= \pm \overline{\boldsymbol{F}}_{p} /\left|\overline{\boldsymbol{F}}_{p}\right|$. The preference given to a positive thrust yields the choice of positive sign for this latter equality, i.e.

$$
\boldsymbol{k}_{r}=\frac{\overline{\boldsymbol{F}}_{p}}{\left|\overline{\boldsymbol{F}}_{p}\right|}
$$

From there, the stabilization of $(\tilde{\boldsymbol{\rho}}, \tilde{\boldsymbol{v}})$ to zero essentially relies on the asymptotic stabilization of the body's frame vector $\boldsymbol{k}$ to the unit "reference" vector $\boldsymbol{k}_{r}$ via the choice of $\omega_{1,2}$. The following theorem, which extends a result in [12] for axisymmetric vehicles, recalls a solution to this problem, and states a convergence and stability property for a class of reference trajectories.

\section{Theorem 1 Assume that}

1) The attack angle along the reference trajectory, denoted as $\alpha_{r}$, is always strictly positive, i.e., $\exists \epsilon>$ $0: \epsilon \leq \alpha_{r}, \forall t$;

2) $\overline{\boldsymbol{F}}_{p}$ does not vanish along the velocity reference trajectory, i.e., $\exists \delta>0: \delta \leq \overline{\boldsymbol{F}}_{p}\left(\boldsymbol{v}_{r, a}(t), \boldsymbol{a}_{r}(t), \mathbf{0}\right), \forall t$, with $\boldsymbol{v}_{r, a}:=\boldsymbol{v}_{r}-\boldsymbol{v}_{w}$.

3) $\boldsymbol{\xi}(\tilde{\boldsymbol{v}})$ makes $(\tilde{\boldsymbol{\rho}}, \tilde{\boldsymbol{v}})=\mathbf{0}$ a locally exponentially stable equilibrium point of the system:

$$
\begin{aligned}
& \dot{\tilde{\boldsymbol{\rho}}}=\boldsymbol{f}(\tilde{\boldsymbol{\rho}}, \tilde{\boldsymbol{v}}) \\
& \dot{\tilde{\boldsymbol{v}}}=\boldsymbol{\xi}
\end{aligned}
$$

Let $T$ be computed according to (19), and the angular velocity $\omega$ be computed according to

$$
\begin{aligned}
& \omega_{1}=\omega_{r, 1}-\left(\nu+\frac{\dot{\gamma}}{\gamma}\right) k_{r, 2} \\
& \omega_{2}=\omega_{r, 2}+\left(\nu+\frac{\gamma}{\gamma}\right) k_{r, 1} \\
& \omega_{3}: \text { given by }(14)
\end{aligned}
$$

with $\boldsymbol{k}_{r}$ defined by (20), $\boldsymbol{\omega}_{r}:=\boldsymbol{k}_{r} \times \dot{\boldsymbol{k}}_{r}, \gamma:=\sqrt{c_{2}+\left|\overline{\boldsymbol{F}}_{p}\right|^{2}}$ with $c_{2}$ any positive constant, and $\nu(\boldsymbol{k}, t)$ any continuous positive real-valued function such that $\inf _{\boldsymbol{k}, t} k_{1}(\boldsymbol{k}, t)>0$.

Then, $(T, \omega)$ ensures local exponential stability of the equilibrium point $(\tilde{\boldsymbol{\rho}}, \tilde{\boldsymbol{v}}, \boldsymbol{k})=\left(\mathbf{0}, \mathbf{0}, \boldsymbol{k}_{r}\right)$ for the system (21a)(16)-(6b).

The proof of this theorem is a straightforward extension of the proof of Proposition 4 in [12]. The main difference between the two results is the smaller set of reference trajectories for which local asymptotic stabilization is proved, due to the first assumption in the theorem that is needed to ensure local uniform exponential convergence of the sideslip angle to zero.

\section{Remarks:}

R.1) Although only local exponential stability is proved, the practical stability domain can be quite large. Indeed, the difficulty to establish a more global stability result comes from the necessity of avoiding the "thin set" of singularities associated with vehicle's orientations such that $\boldsymbol{k} \cdot \boldsymbol{k}_{r}=-1$ and the passage through zero of $\left|\overline{\boldsymbol{F}}_{p}\right|$ and $\alpha$. A complement to the analysis could consist in modifying the control inputs 
near these singularities so as to ensure unconditional wellposedness of the control expressions and prove a "practical" stability result that is more global, with ultimate boundedness of the tracking errors instead of their convergence to zero.

R.2) The assumptions imposed on the reference trajectory are satisfied by a large class of reference trajectories, including standard cruising flight. However, they can be violated when the reference trajectory involves specific aggressive manœuvres. Another complement to this study could thus consists in working out more explicit characterizations of these "good" reference trajectories.

\section{Simulation RESUlts}

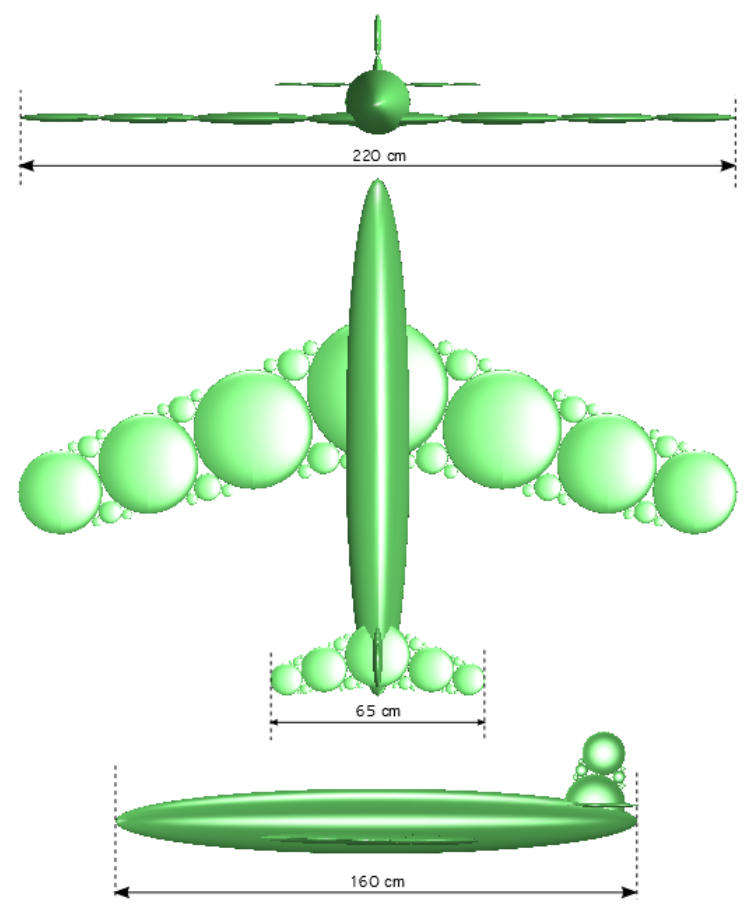

Fig. 2. Simulated fixed-wing UAV composed of an elliptical fuselage and several disk-shaped wings and tails.

We have simulated a fixed-wing UAV sketched in Fig. 2. Size, mass, and inertia specifications of the aircraft are given in Tab. I and Fig. 2. The vehicle's CoM is located at the ellipsoidal fuselage center. Wings and tails (horizontal and vertical) are decomposed into a set of disk-shaped flat surfaces assembled together as shown in Fig. 2. A reason for this decomposition is that a disk, alike an ellipsoid, is an axisymmetric body for which explicit models of the aerodynamic force exerted on it, in the form of functions depending on the disk's center velocity vector (relatively to the ambient air) and the disk's orientation w.r.t. this velocity vector, are available [12]. Using a superposition principle, which does not hold stricto sensu but is commonly used in simulation packages, the resultant aerodynamic force is computed as the sum of the forces applied to all parts (disks+fuselage) taken separately. The resultant parasitic torque is computed in the same manner. Another interesting feature associated with this procedure is that the resultant aerodynamic force is no longer a function of only the aircraft's CoM velocity $\boldsymbol{v}_{a}$ and orientation, contrary to the simplified model used for
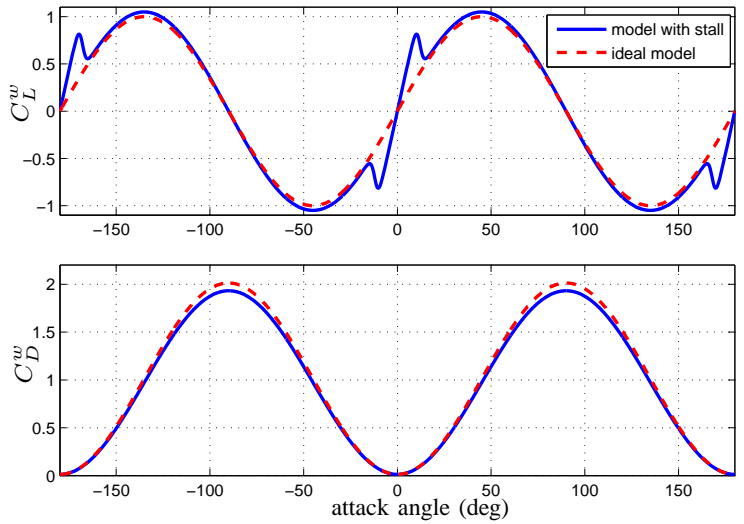

Fig. 3. Approximated analytical model (with stall) and "ideal" model of lift and drag coefficients v.s. attack angle at $R_{e}=160000$ for NACA0018 airfoil.

the control design. It also depends on the aircraft's angular velocity $\boldsymbol{\omega}$, since each disk center velocity is a function of $\boldsymbol{v}_{a}$ and $\boldsymbol{\omega}$. The resulting model is for this reason more complete and precise than the one used for the control design, with the complementary interest that the mismatch between the two models is useful to test the control robustness against modelling errors.

TABLE I

SPECIFICATIONS OF THE SIMULATED FIXED-WING UAV

\begin{tabular}{|c||c|}
\hline Specification & Numerical Value \\
\hline Mass $m[\mathrm{~kg}]$ & 3 \\
Moment of inertia $J\left[\mathrm{~kg} \cdot \mathrm{m}^{2}\right]$ & $\operatorname{diag}(0.033,0.13,0.13)$ \\
Wing total area $\Sigma^{w}\left[\mathrm{~m}^{2}\right]$ & 0.7267 \\
Horizontal tail total area $\Sigma^{t}\left[\mathrm{~m}^{2}\right]$ & 0.1135 \\
Vertical tail total area $\Sigma^{v t}\left[\mathrm{~m}^{2}\right]$ & 0.045 \\
Fuselage total area $\Sigma^{f}\left[\mathrm{~m}^{2}\right]$ & 0.25 \\
\hline
\end{tabular}

In order to simulate the effect of the stall phenomenon, the functions used for the lift and drag coefficients involved in the calculation of the aerodynamic forces applied to the disks composing the wings and tails are chosen so as to closely approximate the measured coefficients of the NACA0018 airfoil at $R_{e}=160000$ (see [11] for complementary details). These functions, and the "ideal" ones defined by (12) and used for the control calculations, are drawn in Fig 3. Discrepancies between these two sets of functions represent another opportunity to test the controller's robustness.

For these simulations, the control variables are composed of the thrust intensity $T$ complemented with a control torque vector $\boldsymbol{\Gamma}$, instead of the angular velocity $\boldsymbol{\omega}$. The angular velocity defined in Theorem 1 is used as a desired angular velocity $\omega^{\star}$, and the role of the torque $\Gamma$ is to make the aircraft's angular velocity $\boldsymbol{\omega}$ closely tracks $\boldsymbol{\omega}^{\star}$. This is achieved by computing the control torque vector as follows

$$
\Gamma=-K_{\omega} J\left(\omega-\omega^{\star}\right)+\omega \times J \omega^{\star}
$$

with $K_{\omega}$ denoting a high-gain positive matrix here chosen equal to $\operatorname{diag}(30,30,30)$. In this calculation, precompensation of the aerodynamic torque and of the reference angular acceleration is deliberately omitted to further test the robustness of the control design against modelling errors. As for the thrust intensity $T$, it is computed according to (19) 

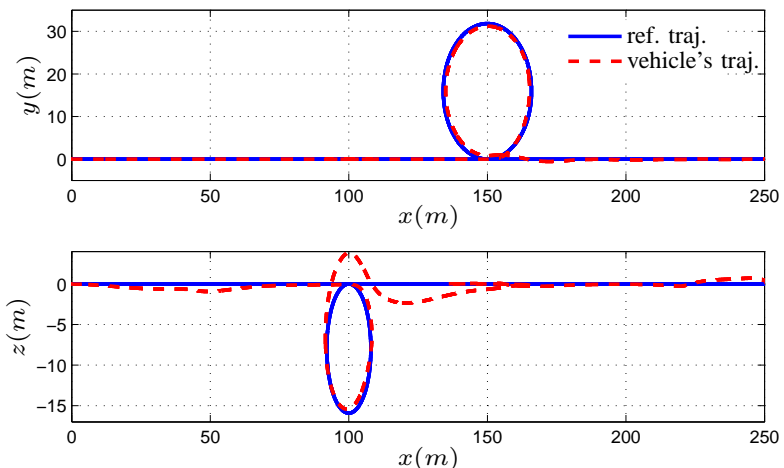

Fig. 4. Reference and vehicle trajectories projected on the horizontal and vertical planes.
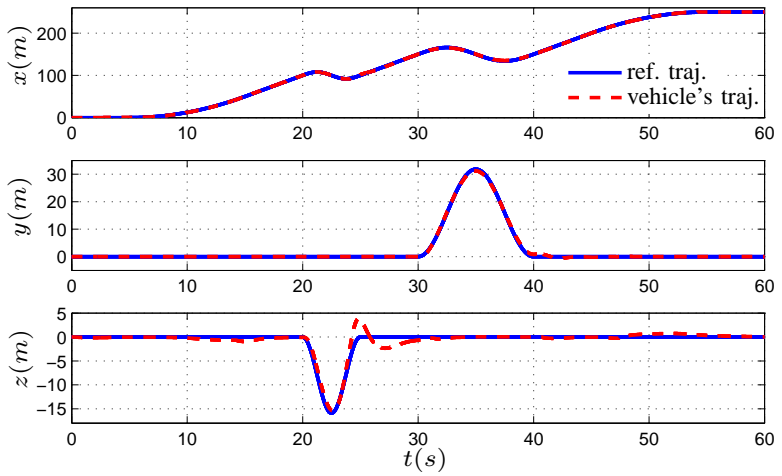

Fig. 5. Reference and vehicle positions versus time.

when the result is positive, and otherwise kept equal to zero in order to be consistent with the positive thrust constraint of standard airplanes and also test the recovery possibilities offered by the control once a positive thrust is again required to track the reference trajectory.

The control objective is the asymptotic stabilization at zero of the position error $\tilde{\boldsymbol{p}}$ with the reference position $\boldsymbol{p}_{r}$ obtained by integration of the reference velocity defined by

$v_{r}(t)=$

$$
\left\{\begin{array}{lr}
(0,0,0) & 0 \leq t<5 \\
(t-5,0,0) & 5 \leq t<15 \\
(10,0,0) & 15 \leq t<20 \\
\left(10 \cos \left(\frac{2 \pi(t-20)}{5}\right), 0,-10 \sin \left(\frac{2 \pi(t-20)}{5}\right)\right) & 20 \leq t<25 \\
(10,0,0) & 25 \leq t<30 \\
\left(10 \cos \left(\frac{2 \pi(t-30)}{10}\right), 10 \sin \left(\frac{2 \pi(t-30)}{10}\right), 0\right) & 30 \leq t<40 \\
(10,0,0) & 40 \leq t<45 \\
(55-t, 0,0) & 45 \leq t<55 \\
(0,0,0) & t \geq 55
\end{array}\right.
$$

The vehicle is initially hovering at the origin of the inertial frame. This velocity profile has been chosen for the vehicle to perform several complex manœuvres. The intermediary control vector $\boldsymbol{\xi}$ is a PID controller determined as follows

$$
\boldsymbol{\xi}(\tilde{\boldsymbol{\rho}}, \tilde{\boldsymbol{v}})=-k_{p}\left(\tilde{\boldsymbol{p}}+k_{i} \boldsymbol{I}_{p}\right)-k_{v}\left(\tilde{\boldsymbol{v}}+k_{i} \frac{d}{d t} \boldsymbol{I}_{p}\right)
$$

with $\tilde{\boldsymbol{\rho}}=\left(\boldsymbol{I}_{p}, \tilde{\boldsymbol{p}}\right), \boldsymbol{I}_{p}$ a numerically calculated saturated integral of $\tilde{\boldsymbol{p}}$ such that $\frac{d}{d t} \boldsymbol{I}_{p}=k_{p z}\left(-\boldsymbol{I}_{p}+\operatorname{sat}_{\Delta}\left(\boldsymbol{I}_{p}+\frac{\tilde{\boldsymbol{p}}}{k_{p z}}\right)\right)$.
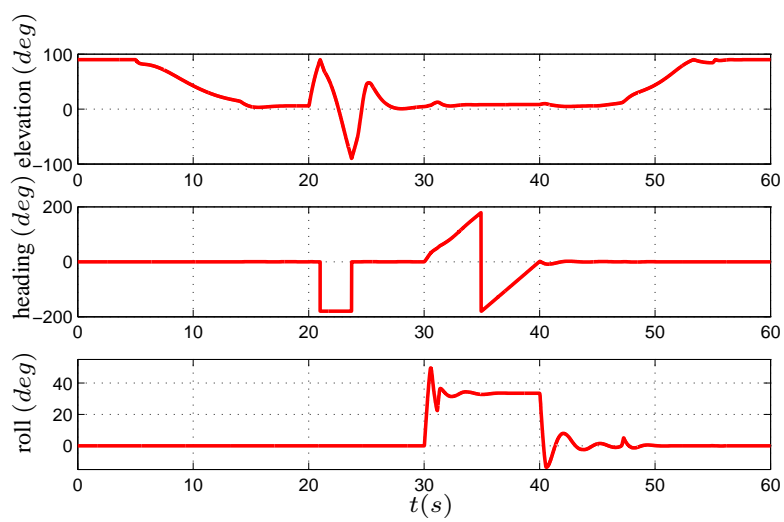

Fig. 6. Vehicle's orientation (elevation, heading, roll angles) versus time.
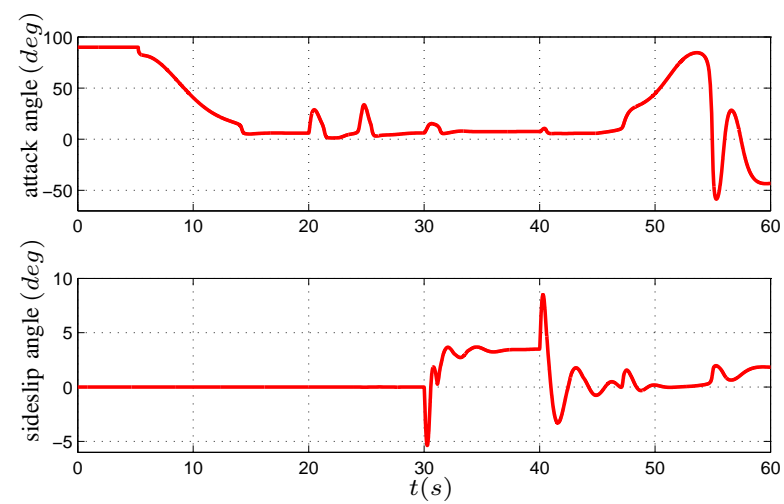

Fig. 7. Attack and sideslip angles versus time.
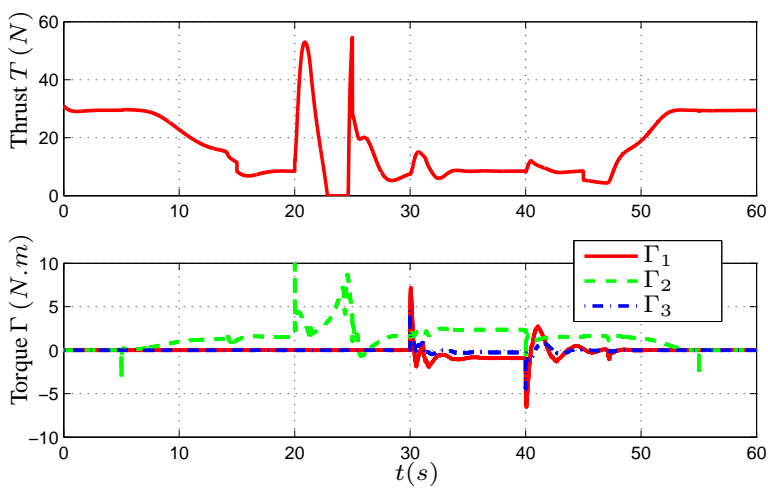

Fig. 8. Thrust intensity and torque inputs versus time.

The gain parameters used for the calculations are $k_{p}=3.24$, $k_{v}=2.5456, k_{i}=0.648, k_{p z}=1, \Delta=4 / k_{i}$. To further assess the robustness of the controller, several parametric errors have been simulated. For instance, we have used $m=$ 2.85 and $J=\operatorname{diag}(0.04,0.1,0.1)$ in the control calculations, instead of the values reported in Table I.

Simulation results are reported next. The projection on the horizontal and vertical planes of the path followed by the vehicle's CoM is shown in Fig. 4. Variations w.r.t. time of the vehicle's position and orientation, of the attack and sideslip angles, and of the thrust intensity and torque inputs are shown in Figs. 5-8. One observes from Figs. 4 and 5 that the vehicle closely follows the reference trajectory.

The transient manœuvre, from hovering to high speed horizontal cruising flight, between $t=5(\mathrm{~s})$ and $t=20(\mathrm{~s})$, 
is smoothly negotiated (see Figs. 6-7). During this transient period the attack angle varies in large proportions, from $90(\mathrm{deg})$ to $5(\mathrm{deg})$. At horizontal cruising speed of $10(\mathrm{~m} / \mathrm{s})$ the attack angle converges to $5.8(\mathrm{deg})$ approximately. The passage through the stall zone (i.e., $\alpha \in[11,14](\operatorname{deg})$ ), when $t \approx 14(s)$, does not jeopardize the system's stability. The transient manœuvre from high speed cruising to hovering, between $t=40(s)$ and $t=55(\mathrm{~s})$, is also performed smoothly.

Concerning the aerobatic stunt induced by the reference velocity variations between $t=20(s)$ and $t=25(s)$, the position error remains small $(<1.2(\mathrm{~m}))$ during the first part of the loop, then grows to $\approx 5(\mathrm{~m})$ at the end of the loop essentially because of the positivity imposed to the thrust intensity during the time interval $t \in[22.7,24.6](s)$ (see Fig. 8-top). Despite this, the vehicle manages to complete the stunt (see Fig. 4-bottom), then catches up and closely track the reference trajectory when the thrust intensity calculated with the control law becomes again positive.

Finally, the turn manœuvre between $t=30(\mathrm{~s})$ and $t=$ $40(s)$ is successfully executed, and a small tracking error $(|\tilde{\boldsymbol{p}}|<1.5(\mathrm{~m}))$ is maintained along a circular horizontal motion. One observes from Fig. 6-bottom and Fig. 7-bottom that the roll angle and the sideslip angle converge to constant values $(\approx 33.8(\mathrm{deg})$ and $\approx 3.5(\mathrm{deg})$, respectively). The fact that the sideslip angle does not converge to zero can be explained by the non-compensation of aerodynamic torques by the control torque. Fig. 7-bottom also shows that, at the time instants $t=30(\mathrm{~s})$ and $t=40(\mathrm{~s})$, the sideslip angle has short bursts essentially because of the discontinuity of the reference acceleration at these time instants. Finally, one can also observe from Fig. 7-top that the attack angle varies in large proportions during the hovering manœuvre after $t=54(s)$. This is coherent with the fact that the determination of this angle is ill-conditioned when the body's air-velocity $\boldsymbol{v}_{a}$ vanishes.

\section{CONCLUDING REMARKS}

A novel approach to the automatic control of scale model airplanes, which extends earlier studies by the authors on the control of VTOL and axisymmetric vehicles, has been presented. With respect to other studies on the subject, and in order to extend the control operating domain, a salient feature of the approach is the use of simple analytical models of the aerodynamic forces applied to the aircraft that cover the whole range of attack angles. Implementation aspects (actuation and sensors related issues, in particular) and experiments conducted on physical scale model airplanes will be the subject of forthcoming studies.

\section{ACKNOWLEDGEMENT}

This work was supported by the French Agence $\mathrm{Na}$ tionale de la Recherche through the ANR ASTRID SCAR project "Sensory Control of Aerial Robots" and by the Chaire d'excellence en robotique RTE-UPMC "Mini-drones autonomes".

\section{APPENDIX}

Proof of Proposition 2:

$$
\begin{aligned}
& \text { From (2), (3), and (5), } \\
& \qquad \begin{aligned}
F_{a, 2} & =-\eta_{a}\left|\boldsymbol{v}_{a}\right| C_{D} v_{a, 2}-\eta_{a}\left|\boldsymbol{v}_{a}\right|^{2} C_{L} i_{a, 2} \\
& =-\eta_{a}\left|\boldsymbol{v}_{a}\right| C_{D} v_{a, 2}-\eta_{a}\left|\boldsymbol{v}_{a}\right|^{2} k_{a, 1} \frac{C_{L}}{k_{a, 1}} i_{a, 2} \\
& =-\eta_{a}\left|\boldsymbol{v}_{a}\right| C_{D} v_{a, 2}+\eta_{a}\left|\boldsymbol{v}_{a}\right| v_{a, 1} \frac{C_{L}}{k_{a, 1}} i_{a, 2}
\end{aligned}
\end{aligned}
$$

where the last equality follows from (1). This equality can in turn be written as

$$
\begin{aligned}
F_{a, 2} & =-\eta_{a}\left|\boldsymbol{v}_{a}\right| C_{D} v_{a, 2}+\eta_{a}\left|\boldsymbol{v}_{a}\right| k_{a, 2} v_{a, 1} \frac{C_{L}}{k_{a, 1}} \frac{i_{a, 2}}{k_{a, 2}} \\
& =-\eta_{a}\left|\boldsymbol{v}_{a}\right| C_{D} v_{a, 2}-\eta_{a} v_{a, 2} v_{a, 1} \frac{C_{L}}{k_{a, 1}} \frac{i_{a, 2}}{k_{a, 2}}
\end{aligned}
$$

so that (9) is satisfied with

$$
a\left(\boldsymbol{k}_{a}\right)=\eta_{a} C_{D}\left(\boldsymbol{k}_{a}\right), \quad b\left(\boldsymbol{k}_{a}\right)=-\eta_{a} \frac{C_{L}\left(\boldsymbol{k}_{a}\right)}{k_{a, 1}} \frac{i_{a, 2}}{k_{a, 2}}
$$

The function $a$ is continuous and strictly positive since $C_{D}$ also satisfies these properties and $\eta_{a}>0$ is constant. Since $C_{L}$ is smooth and vanishes identically at $\alpha=0, \pi$, which corresponds to $k_{a, 1}=0$, the function $\frac{C_{L}\left(\boldsymbol{k}_{a}\right)}{k_{a, 1}}$ can be defined by continuity at these points. There remains to show that $\frac{i_{a, 2}}{k_{a, 2}}$ can be defined by continuity at $k_{a, 2}=0$. This follows from assumption of smoothness of $\boldsymbol{i}_{a}$ w.r.t. $\boldsymbol{k}_{a}$ and to the fact that $k_{a, 2}=0$, implies $i_{a, 2}=0$.

\section{REFERENCES}

[1] S. Bertrand, H. Piet-Lahanier, and T. Hamel. Contractive model predictive control of an unmanned aerial vehicle model. In 17th IFAC Symp. on Automatic Control in Aerospace, volume 17, 2007.

[2] S. Bouabdallah and R. Siegwart. Backstepping and sliding-mode techniques applied to an indoor micro quadrotor. In IEEE International Conference on Robotics and Automation, 2005.

[3] J. Hauser, S. Sastry, and G. Meyer. Nonlinear control design for slightly non-minimum phase systems: Application to V/STOL. Automatica, 28:651-670, 1992.

[4] M.-D. Hua, T. Hamel, P. Morin, and C. Samson. Introduction to feedback control of underactuated vtol vehicles. IEEE Control Systems Magazine, pages 61-75, 2013.

[5] M.D. Hua, T. Hamel, P. Morin, and C. Samson. A control approach for thrust-propelled underactuated vehicles and its application to vtol drones. IEEE Transactions on Automatic Control, 54:1837-1853, 2009.

[6] A. Isidori, L. Marconi, and A. Serrani. Robust autonomous guidance: an internal-model based approach. Springer Verlag, 2003.

[7] H. J. Kim, D. H. Shim, and S. Sastry. Nonlinear model predictive tracking control for rotorcraft-based unmanned aerial vehicles. In American Control Conference, pages 3576-3581, 2002.

[8] L. Marconi, A. Isidori, and A. Serrani. Autonomous vertical landing on an oscillating platform: an internal-model based approach. Automatica, $38: 21-32,2002$.

[9] E. Muir. Robust flight control design challenge problem formulation and manual: The high incidence research model (hirm). In Robust Flight Control, A Design Challenge (GARTEUR), volume 224 of Lecture Notes in Control and Information Sciences, pages 419-443. Springer Verlag, 1997.

[10] D. Pucci. Flight dynamics and control in relation to stall. In American Control Conf. (ACC), pages 118-124, 2012.

[11] D. Pucci. Towards a unified approach for the control of aerial vehicles. $\mathrm{PhD}$ thesis, Université de Nice-Sophia Antipolis and "Sapienza" Universita di Roma, 2013.

[12] D. Pucci, T. Hamel, P. Morin, and C. Samson. Nonlinear feedback control of axisymmetric aerial vehicles. Arxiv, page 17p, 2014.

[13] S. N. Singh and A. Schy. Output feedback nonlinear decoupled control synthesis and observer design for maneuvering aircraft. International Journal of Control, 31(31):781-806, 1980.

[14] Q. Wang and R.F. Stengel. Robust nonlinear flight control of high-performance aircraft. IEEE Transactions on Control Systems Technology, 13(1):15-26, 2005.

[15] R. Xu and U. Ozguner. Sliding mode control of a class of underactuated systems. Automatica, 44:233-241, 2008. 